ance to this treatment but was improved by IVIG. There was a temporal relationship between neurological and renal improvement and IVIG, and this could reflect a common pathogenesis. Recognition of such coexisting disorders provides further evidence of an immune dysfunction in CIDP.

$$
\begin{array}{r}
\text { A P FĖVE } \\
\text { D SMADJA } \\
\text { G RANCUREL } \\
\text { J M LEGER } \\
\text { Service de Neurologie, } \\
\text { Pr P Brunet, } \\
\text { Hôpital SALPETRIERE, Paris } \\
\text { Service de Neurologie, } \\
\text { Pr A Guillard } \\
\text { Hopital TENON, Paris, France }
\end{array}
$$

Correspondence to: Dr A P Fève, Service de Neurologie, Hôpital TENON, 4 rue de la Chine, 75020 Paris, France.

1 Dyck PJ, Arnason B. Chronic inflammatory demyelinating polyradiculoneuropathy. In: Dyck PJ, Thomas PK, Lambert EM, Buge R, Dyck PJ, Thomas PK, Lambert EM, Buge R,
eds. Peripheral neuropathy vol 2. Philadelphia eds. Peripheral neuropathy vol 2. Philadelphia

2 Korn Lubetzki I, Abramsky O. Acute and chronic demyelinating inflammatory polyradiculoneuropathy. Association with autoimmune diseases and lymphocytes response to human neuritogenic protein. Arch Neurol 1986;43:604-8.

3 Green D. Circulating anticoagulants. Med Clin $N$ Amer 1972;56:146-51.

4 Berger JR, Rosenfeld WE, Sheremata WA, et al. Chronic inflammatory polyradioculoneuropathy complicated by factor VIII antibody. Neurology 1983;33:1224-6.

5 White AS, Burke JF. Membranous glomerulonephritis associated with chronic progressive demyelinating neuropathy. Neurology 1987; 37:342-5.

Acute stage Bell's palsy and narrowing of the palpebral fissure caused by drooping of the eyebrow and the upper eyelid

Widened palpebral fissure on the affected side has been regarded as one of the hallmarks of peripheral facial palsy. ${ }^{12}$ The palpebral fissure of the affected eye may, however, be narrower than that of the healthy eye..$^{3-5}$

Twenty five patients with Bell's palsy in the acute stage were examined at our outpatient

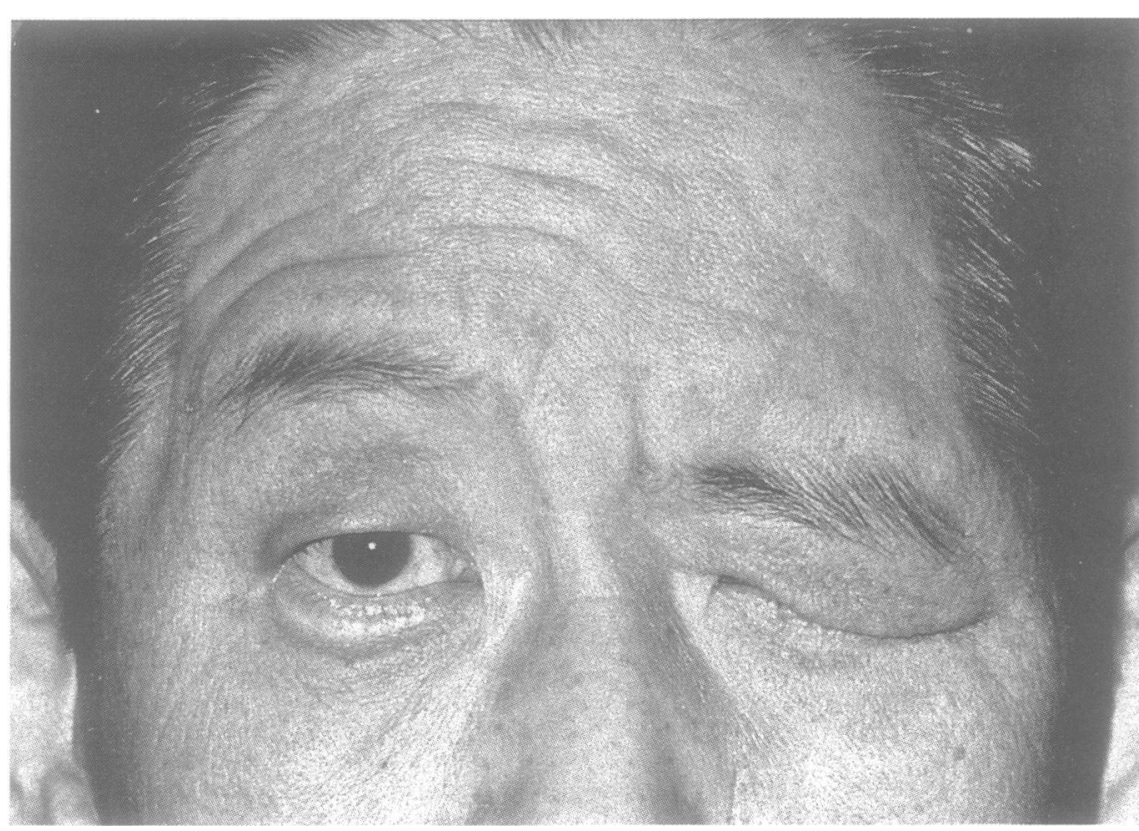

Figure Patient looking straight ahead. Narrowing of the palpebral fissure is seen on the left side. other neurological signs were observed in these patients. Ten patients had a narrowed palpebral fissue rather than widened palpebral fissure on the affected side looking straight ahead. Their ages ranged from 32-59 years with a mean of 46.8 years. Seven patients were men and three women, four being diabetic. All 10 patients complained of a drooping of the upper eyelid on the affected side when opening their eyes. All had a furrowless forehead and the corner of the mouth drooped on the affected side, both characteristics of peripheral facial palsy. They were unable to close the affected eye tightly, and five patients had lagophthalmos (incomplete closure). The position of the eyebrow and the upper eyelid on the affected side was lower and the width between their lower margins was shorter than on the non-affected side (fig). The patients experienced difficulty in elevating the eyebrow and the upper eyelid when gazing upwards. The position of the eyes was intact, however, and the extraocular movements were full. The pupils were round, equal in size and reactive to light.

All the patients had CT or MRI brain scans. All were free from intracranial lesions. Neuroelectrophysiological examinations were performed in five patients and in these patients the distal latency of the facial nerve was prolonged and surface electromyogram of the frontal muscle showed decreased activity on the affected side.

The principal muscle involved in opening the upper eyelid and maintaining normal eyelid posture is the superior palpebral levator, which is innervated by the oculomotor nerve. Two accessory muscles are Müller's muscle via the oculosympathetic pathway and the frontal muscle by the facial nerve. The upper eyelid is indirectly elevated by the attachments of the frontal muscle into the eyebrow with the superior orbital portions of the orbicularis oculi muscle. 6 Thus it is possible that the weakness of the frontal muscle causes drooping of the eyebrow and the upper eyelid, resulting in narrowing of the palpebral fissure. This phenomenon might be more noticeable in Japanese than in

clinic from January 1990 to May 1991. No 
abducens nerve palsy resolved gradually over 4 months.

The patient has one healthy son (age 8) and an unaffected mother, but his father and brother (the patient's only sibling) had experienced cranial nerve palsies in the past. At the age of $39(5 / 89)$, the brother developed a right abducens nerve palsy that resolved over 6 weeks. Three years previously he had had a myocardial infarction and subsequently required a 5-vessel coronary artery bypass. The patient's father, an only child, experienced a left Bell's palsy at age 49 and a left abducens nerve palsy at age $59(7 / 84)$. At age 44 he was found to have 3 vessel disease by cardiac catheterisation. Both brother and father had normal neuroophthalmological examinations in January 1991.

All three affected family members had a thorough haematologic evaluation for disorders predisposing to thrombosis including assays for cardiolipin antibody, the Lupus anticoagulant (Russel viper venom time), prothrombin, partial thromboplastin, fibrinogen, fibrin split products, plasminogen, antithrombin III, protein $\mathrm{C}$, and protein $\mathrm{S}$. These tests were all normal or negative.

The familial occurrence of recurrent cranial nerve palsies was first described by Stone in 1950.' Subsequently, several other families have been reported. ${ }^{23}$ The aetiology of the cranial nerve palsies remains unclear despite extensive evaluation including glucose tolerance tests, serologic testing, edrophonium (Tensilon) tests, cerebrospinal fluid analysis, single fibre electromyography, CT scan, and cerebral angiography.

Recently, haematological disorders predisposing to thrombosis have been associated with numerous neurological and systemic manifestations in young adults, including cerebral and myocardial infarction, deep venous thrombosis and migraine. ${ }^{4}$ Ophthalmic consequences (retinal arterial and venous occlusion, vitreous hemorrhage and ischaemic optic neuropathy) have also been reported in these patients. ${ }^{5}$ Thrombosis in these disorders may be caused by a deficiency of one or more proteins integral to the regulation of clot formation (antithrombin III, protein C, protein S, fibrinogen) or by the presence of antiphospholipid antibodies (cardiolipin antibody, Lupus anticoagulant) that interfere with phospholipid dependent activation complexes (prothrombinase) essential to clot regulation. These tests were not available for the evaluation of previously reported patients with the syndrome of familial recurrent cranial nerve palsy. We believe that the pathophysiology of nerve damage in this syndrome is vasculopathic because several of our patients had other evidence of vascular disease and vasculopathic disorders (diabetes, hypertension, coronary artery disease). These vasculopathic disorders are associated with cranial nerve palsies that present and improve in a similiar manner. Our patients, however, had no laboratory evidence of antiphospholipid antibodies or deficiencies of coagulation cascade proteins and thus no identifiable predisposition to thrombosis. Familial recurrent cranial nerve palsy may be caused by an as yet undefined vasculopathic process.

KARL C GOLNIK Medical University of South Carolina Medical University of South Carolina,
Charleston, South Carolina, 29425, USA The Wilmer Institute The fohn Hopkins Hospital, Baltimore, Correspondence to: Dr Miller.
1 Stone TT. Peripheral facial palsy. Multiple attacks in three brothers. FAMA 1950; 143:1154-5.

2 Currie S. Familial oculomotor palsy with Bell's palsy. Brain 1970;93:193-8.

3 Aldrich MS, Beck RW, Albers JW. Familial recurrent Bell's palsy with ocular motor recurrent Bell's palsy with ocular

4 Comp PC. Hereditary disorders predisposing to thrombosis. Prog Hemostas Thromb 1986; 8:71-102.

5 Kleiner RC, Najarian LV, Schatten S, et al. Vaso-occlusive retinopathy associated with anti-phospholipid antibodies (lupus anticoagulant retinopathy). Ophthalmology 1989; 96:896-904.

\section{Transient amnesia heralding brain stem} infarction

Transient global amnesia (TGA) is a syndrome of acute, transient memory disturbance with severe anterograde and retrograde amnesia but no neurological signs and preservation of personal identity. Vascular, epileptic, migrainous and hypoglycaemic causes have been implicated in its aetiology. ${ }^{12}$

A recent case control study has shown that most episodes of TGA are not associated with risk factors for ischaemic cerebral disease. ${ }^{34}$ Whilst ischaemia may lead to amnesic syndromes, previous reports of apparent TGA as a manifestation of transient cerebral ischaemia, have rarely included witnessed accounts of the ischaemic episode.

A 46 year old man presented having had five episodes of vertigo, each lasting for several hours, over the previous six months. Examination between the attacks was normal apart from mild hypertension and marked symptoms of anxiety. When seen as an outpatient routine investigations were unremarkable. There was no evidence of a peripheral labyrinthine disturbance and the brainstem evoked potentials, CT and MRI scans were normal. He was referred for a psychiatric opinion. There was no previous psychiatric history, although over the previous months, following his divorce and threats to his job, he had become anxious about his future. He was known to have drunk heavily in the past but had not done so in the previous year.

Three months later, when attending the outpatient department, he suddenly complained of memory loss. This episode lasted for 5 hours during which time psychometric assessment illustrated the fluctuating nature of the memory deficit. He initially appeared agitated and bewildered, repeatedly asking the date, where he was and why he was there. He was alert and could recall his name and date of birth. One hour later neuropsychological assessment showed that he performed satisfactorily on a relatively easy, three choice recognition memory test comprising coloured photographs. His ability to recognise and name famous faces was also within the average range. Further testing was not possible as he began to complain of blurred vision and loss of memory. On questioning during the next half an hour he appeared to experience total lapses of memory interchangeably with reasonable accounts of his present circumstances. Two hours later there was a profound anterograde amnesia and patchy retrograde amnesia. There were no other abnormal neurological signs. An EEG performed at the height of his symptoms showed a few slow wave transients in the frontotemporal region. On the following morning (that is, 14 hours later) his ability to recall new information had returned to normal.
There remained, however, an amnesic gap for a short period of his train journey to the hospital on the previous day.

Three days later, while still in hospital, he had a brief episode of dysarthria, numbness of the tongue, weakness and clumsiness of the left arm. There were no neurological abnormalities when he was examined shortly after this episode, which was diagnosed as a transient ischaemic attack. The next day he had a further transient episode of dysarthria and right hemiparesis, again with no signs on examination immediately after the event

Twenty four hours later he became drowsy and developed periodic ventilation. $\mathrm{He}$ had gaze evoked nystagmus to the right with delayed adduction of the right eye on leftward gaze, preserved upgaze with upbeat nystagmus, constricted pupils, right facia weakness, diminished pharyngeal reflex, slow tongue movements and a dense right hemiparesis. CT scan showed low attenuation in the right inferior cerebellar hemisphere and right occipital lobe consistent with infarction. MRI confirmed the diagnosis of brainstem stroke, with extensive high signal at the right cerebellar hemisphere, brain stem and medial part of right occipital lobe suggestive of occlusion of right posterior inferior cerebellar artery and right posterior cerebral artery.

There was gradual neurological recovery over two months. He often appeared distressed when discussing the future during this period but his mental state was otherwise considered to be normal and he performed satisfactorily on both verbal and visual recognition memory tests.

This patient presented with an episode of amnesia with features of TGA. ${ }^{3}$ There was a witnessed attack of definite amnesia, resolving within 24 hours, without disturbance of consciousness, focal neurological signs, epileptic features or a recent head injury. During the attack the patient developed a clear anterograde amnesia with disturbance of long term memory without loss of persona identity, complex cognition or language. ${ }^{5} \mathrm{He}$ also exhibited the phenomenon of "metamemory", an awareness that memories ough to be readily recalled. Following recovery a small gap of retrograde amnesia remained. The only neurological symptom was transiently blurred vision but examination was normal during the episode.

This case serves to emphasise that transient amnesia resembling TGA may occur as a manifestation of transient ischaemic attacks in the vertebrobasilar territory and that there is a risk of subsequent major ischaemic deficit.

R S HOWARD
R FESTENSTEIN
J MELLERS
L D KARTSOUNIS
M RON
The National Hospital for Neurology and
Neurosurgery, London, UK

Correspondence to: Dr Howard, The National Hospital for Neurology and Neurosurgery, Queen Square, London WCIN 3BG, UK.

1 Markowitsch HJ (ed). Transient global amnesia and related disorders. Toronto: Hogrefe and Huber, 1990.

2 Fisher CM, Adams RD. Transient global amnesia. Acta Neurol Scand 1964;40:1-83.

3 Hodges JR, Warlow CP. Syndromes of transient amnesia: Towards a classification. $\mathfrak{f}$ Neurol amnesia: Towards a classification. $\mathcal{F}$

4 Hodges JR, Warlow CP. The aetiology of transient globalamnesia. A case control study of sient globalamnesia. A case control study of 1990;113:639-57.

5 Hodges JR, Ward CD. Observations during transient global amnesia. Brain 1989;112 595-620. 